\title{
Nanofabrication of gallium nitride photonic crystal light-emitting diodes
}

\author{
Ali Z. Khokhar ${ }^{\mathrm{a}}$, Keith Parsons ${ }^{\mathrm{b}}$, Graham Hubbard ${ }^{\mathrm{b}}$, Faiz Rahman ${ }^{\mathrm{a}, *}$, Douglas S. Macintyre ${ }^{\mathrm{a}}$, Chang Xiong ${ }^{\mathrm{c}, 1}$, \\ David Massoubre ${ }^{c}$, Zheng Gong ${ }^{c}$, Nigel P. Johnson ${ }^{a}$, Richard M. De La Rue ${ }^{a}$, Ian M. Watson ${ }^{c}$, \\ Erdan Gu ${ }^{c}$, Martin D. Dawson ${ }^{\mathrm{c}}$, Steve J. Abbott ${ }^{\mathrm{b}}$, Martin D.B. Charlton ${ }^{\mathrm{d}}$, Martin Tillin ${ }^{\mathrm{e}}$ \\ ${ }^{a}$ Department of Electronics and Electrical Engineering, University of Glasgow, Oakfield Avenue, Rankine Building, Glasgow G12 8LT, United Kingdom \\ ${ }^{\mathrm{b}}$ MacDermid Autotype Ltd, Grove Road, Wantage OX12 7BZ, United Kingdom \\ ' Institute of Photonics, University of Strathclyde, Wolfson Centre, 106 Rottenrow, Glasgow G4 0NW, United Kingdom \\ ${ }^{\mathrm{d}}$ School of Electronics and Computer Science, University of Southampton, Southampton SO17 1BJ, United Kingdom \\ e Sharp Laboratories of Europe Ltd, Oxford Science Park, Oxford OX4 4GB, United Kingdom
}

\section{A R T I C L E I N F O}

\section{Article history:}

Received 22 October 2009

Received in revised form 6 January 2010

Accepted 3 February 2010

Available online $\mathrm{xxxx}$

\section{Keywords:}

Light-emitting diodes

Photonic crystals

Nanolithography

GaN dry-etching

\begin{abstract}
A B S T R A C T
We describe a comparison of nanofabrication technologies for the fabrication of 2D photonic crystal structures on GaN/InGaN blue LEDs. Such devices exhibit enhanced brightness and the possibility of controlling the angular emission profile of emitted light. This paper describes three nano lithography techniques for patterning photonic crystal structures on the emitting faces of LEDs: direct-write electron beam lithography, hard stamp nanoimprint lithography and soft-stamp nanoimprint lithography with disposable embossing masters. In each case we describe variations on the technique as well as its advantages and disadvantages. Complete process details have been given for all three techniques. In addition, we show how high performance GaN dry etch techniques, coupled with optical process monitoring can transfer resist patterns into underlying GaN material with high fidelity.
\end{abstract}

(c) 2010 Elsevier B.V. All rights reserved.

\section{Introduction}

Light-emitting diode (LED) technology is undergoing extremely rapid developments at this time. Many academic, government and industrial laboratories across the world are working on improving their efficiency and lifetime while at the same time bringing down their cost so that they can replace more conventional light sources in a variety of applications $[1,2]$. Nitride semiconductors from the GaN family provide the current materials technology for emitters in the blue and green, and achieve external quantum efficiencies up to $\sim 60 \%$ for packaged blue LED lamps [3]. Phosphide semiconductors meanwhile continue to offer the highest performance at red visible wavelengths. LED-based illumination has been taking over in sectors such as traffic lights and automobile lighting and is now set to make bold strides into areas such as car headlights and street lighting. Electronics manufacturers are also particularly interested in utilising LEDs for backlights in LCD televisions and later on for general purpose room lighting applications. The opportunities to develop LEDs with tailored angular emission profiles for

\footnotetext{
* Corresponding author. Tel.: +440141330 2091; fax: +44 01413304907.

E-mail address: f.rahman@elec.gla.ac.uk (F. Rahman).

1 Present address: School of Physics and State Key Laboratory for Artificial Microstructures and Mesoscopic Physics, Peking University, Beijing 100871, People's Republic of China.
}

more specialised applications, including backlights, projectors and fibre optic communications, are also significant. Harbers et al. give a recent discussion of the potential for LED solutions in the first two application areas [4]. Incorporation of a photonic crystal structure on the emitting face of LEDs is now recognised as perhaps the most important technology for adapting LEDs to various application requirements. The fabrication techniques for photonic crystal (PhC) and photonic quasi-crystal (PhQC) LEDs described in this paper will have their first commercial impact in applications requiring increased brightness and optimised angular emission profiles as prime considerations.

All inorganic LEDs generate light inside a high-index medium, and this factor limits the efficiencies attained by simple planar device architectures. This feature arises due to total internal reflection of a large fraction of light within the high refractive index GaN epitaxial layers. As a result, much of the light remains confined as trapped modes inside these layers. The numerical extraction efficiency is only $\sim 4 \%$ for GaN-based devices coupling directly into air, but can be increased up to $\sim 10 \%$ by encapsulation with a transparent polymer [5]. Several other techniques have been developed to extract even more of the internally trapped radiation. These include random surface roughening [6], use of corrugated or patterned substrates $[7,8]$ and patterning the LED surface with a photonic crystal structure [9-12]. PhC and PhQCs function by coupling modes trapped in high-index material into modes which 
can escape. In this context, the possibility of fabricating thin device structures by laser lift-off (LLO) processing of GaN-based epitaxial structures grown on sapphire is very important [13]. This is because such techniques remove sapphire and thus modes trapped in sapphire and also maximise the interaction between trapped modes in GaN and the etched PhC structures. The interaction of guided modes with a $\mathrm{PhC} / \mathrm{PhQC}$ grating can be maximised when the total GaN thickness is reduced to only a few microns [3]. Furthermore, heat dissipation is also greatly improved over conventional devices, as heat transport through sapphire substrate is replaced by much more efficient heat transfer through metallic back contacts. LLO was recently used in the fabrication of thin GaN-based PhC LEDs with a record extraction efficiency exceeding 70\% for non-encapsulated devices [14].

In addition to improvement of extraction efficiencies, $\mathrm{PhC}$ and PhQC patterning allows tailored modifications to the polar radiation characteristics of LEDs from the near-Lambertian pattern obtained using planar non-encapsulated devices. Much attention has been focussed on enhancing emission in directions close to the surface normal, as desirable in étendue-limited applications $[3,15]$. However, it is also possible to design $\mathrm{PhC}$ and $\mathrm{PhQC}$ patterns which cause preferential light emission at large angles to the surface normal, as desirable for certain forms of display backlights; specifically direct illumination of flat panels [16]. Here highly homogeneous illumination of the applications plane is critical. PhQC patterns departing from traditional crystallographic symmetry offer major advantages over their regular PhC counterparts by virtue of the azimuthal uniformity of emission $[3,16,17]$.

All implementations of PhC and PhQC patterns in visible LEDs require a sub-micron patterning capability, and a brief overview of techniques available is included in Ref. [3]. In this paper, we report on direct-write electron beam lithography (EBL), and two different embodiments of nanoimprint lithography (NIL). NIL techniques have superior potential for mass production, and Watts et al. reviewed currently available fabrication equipment and economic considerations [18]. In all process flows, the PhC/PhQC pattern is first transferred into a mask layer, and must then be transferred into the semiconductor material by a dry etch process, allowing good control over the hole depth and sidewall profile. Currently most $\mathrm{PhC}$ and PhQC designs assume vertical sidewalls, and etch processes must therefore offer a high degree of anisotropy to approximate this idealised geometry in the real device. For GaN-based devices, conventional and thin LEDs made by LLO have different constraints on hole depth, as can be understood with reference to the typical $p-i-n$ device structure on sapphire shown in Fig. 1 . The topmost $p$-type layers of commercial LED wafers above the active region are normally kept to a thickness of $\sim 150 \mathrm{~nm}$. Therefore in devices with the conventional (so-called $p$-up) geometry, the PhC/PhQC hole etch depth cannot exceed this value without catastrophic shorting of the junction, and/or etch damage to the active region. In contrast, in thin GaN devices made by LLO processing, the depth of holes etched into $n$-type GaN can be significantly greater, as illustrated by published device results $[14,19]$.

This paper presents results from the integrated development and optimisation of patterning and dry etch nanofabrication technologies, with particular reference to GaN-based LEDs. We show results on the processing of as-grown GaN LED wafers on sapphire, corresponding to process flows for conventional p-up LEDs. Fig. 1 shows the structure of a typical device. However, the processing techniques developed are applicable to thin GaN devices made by LLO routes, as well as to LEDs fabricated from non-nitride materials. The results to date have been obtained using 2-inch sapphire based wafers. However, the techniques developed are applicable to GaN device structures on larger sapphire wafers [20], and lowercost large silicon substrates [21]. Both these approaches are being actively pursued to reduce the epitaxy-related cost component of

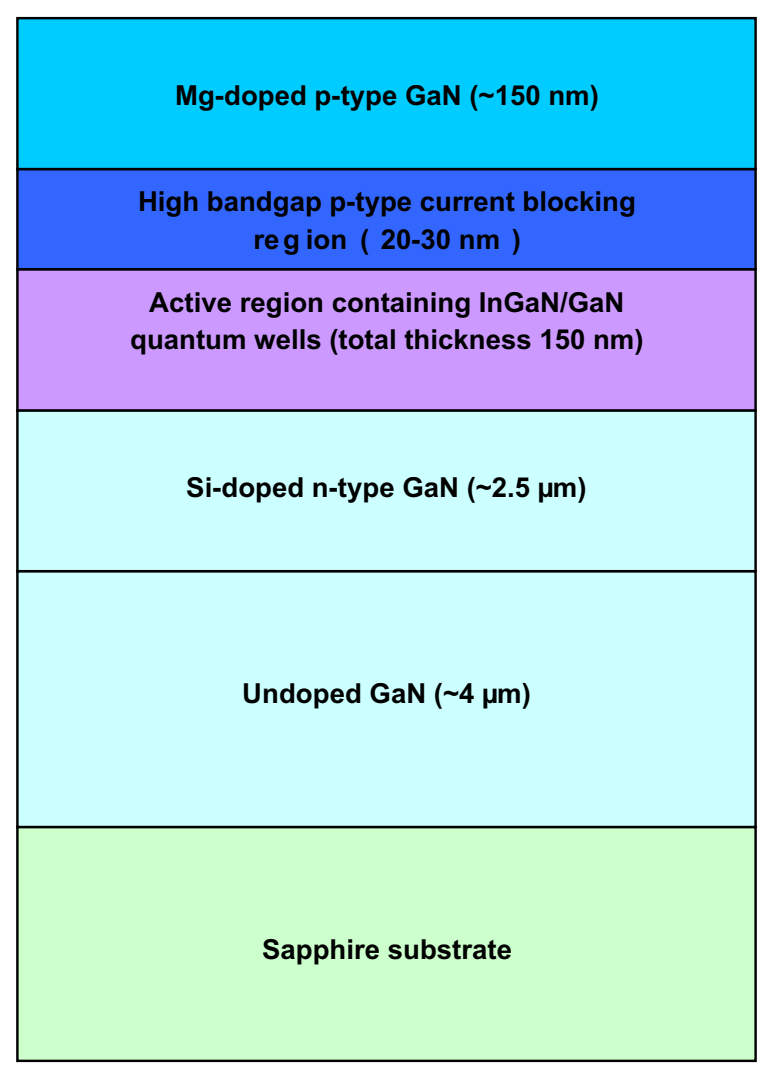

Fig. 1. Structure of a typical epitaxial GaN blue LED wafer material showing the positions and thicknesses of various layers.

GaN LEDs. Our specific motivation concerned the development of PhQC LEDs for direct flat panel backlighting. Results on the performance characteristics of devices made by optimised fabrication routes will be reported elsewhere.

\section{PhC nanofabrication with direct-write e-beam lithography}

Direct-write e-beam lithography has been used for many years to write nanometre-scale features on semiconductors. Photonic crystal structures consisting of arrays of holes, several hundred nanometres in diameter and spaced a few hundred nanometres apart, can be, and are, easily written by e-beam lithography. This technique, though not suitable for commercial production, is very well suited to R\&D operations as no masks or stamps need to be prepared. We have carried out PhC patterning using the directwrite technique with a view to explore the suitability of various resists for defining these structures.

In this work we patterned a 12 -fold photonic quasi-crystal design [22] with different hole diameters and pitch on GaN LED epi-layer wafers. It has been proved that by changing the hole diameter and/or pitch the emission profile of photonic crystal LEDs could be modified [23]. Various e-beam resists such as Hydrogen SilsesQuioxane (HSQ) - a silica-based resist, NEB31 - a chemically-amplified resist from Sumitomo, ZEP-520A a non chemically-amplified resist from Nippon Zeon and PolyMethyl MethAcrylate (PMMA) were used to write PhC patterns. HSQ and NEB31 are negative tone resists whereas ZEP-520A and PMMA are positive tone resists. Leica VB6 e-beam tool was used to write patterns on commercial 2" GaN LED epi-layer wafers - sourced form Lumi-innovations. VB6 is a very high resolution machine and can produce e-beam spot sizes down to around $4 \mathrm{~nm}$ diameter on a design grid of $1.25 \mathrm{~nm}$. 
GaN LED epi-layer wafers were cleaned with acetone, methanol, isopropanol and purified water before spinning on resist. All GaN samples were coated with $200 \mathrm{~nm}$ thick resist. It was found that $30 \mathrm{~nm}$ of an Aluminium (Al) layer on top of the resist mask gives very high quality patterns - Al layer acts as a discharge layer for e-beam lithography. It was also found that HSQ has a number of advantages over other e-beam resists such as:

1. HSQ acts as a direct hard mask for dry-etching. While using HSQ there is no need to use a separate metal hard mask. We found no significant change in the $200 \mathrm{~nm}$ HSQ mask during the etching of up to $300 \mathrm{~nm}$ of GaN LED epi-layer wafer. With other ebeam resists, such as ZEP-520A or PMMA, we needed to use a metal hard mask such as a $\mathrm{NiCr}$ mask on top of the e-beam resist [24].

2. It is not sensitive to white light. We deposited $30 \mathrm{~nm}$ of Al layer on the top of resist to act as a discharge layer for e-beam writing. The Al layer was deposited by using a Plassys e-beam evaporator tool. It was found that NEB31 resist was exposed during a similar deposition of Al layer.

3. HSQ can be easily removed from GaN LED epi-layer wafers using buffered HF.

With HSQ, the best e-beam dose was found to be $700-800 \mu \mathrm{C} /$ $\mathrm{cm}^{2}$ at $100 \mathrm{kV}$ e-beam accelerating voltage. This was determined by using a dose estimation matrix. The hole size can be varied by changing the e-beam dose [25]. The criterion chosen for the best e-beam dose was the achievement of exactly the same size hole diameter in the HSQ resist as was provided in simulated designs. Each sample contained an array of $4 \times 4$ patterns together with four alignment markers. These alignment markers were used to fabricate $n$ and $p$ contact after dry-etching of the photonic crystal pattern. Each pattern had dimensions of $300 \times 300 \mu \mathrm{m}$. Samples also contained blank devices. These blank devices were intended to compare LEDs with and without PhQC pattern. After e-beam writing the samples were developed in Tetra Methyl Ammonium
Hydroxide (TMAH) resist developer and inspected under an optical microscope. Patterns in HSQ were transferred to the epi-layer by a chlorine-based dry etch process that is described in Section 5. After dry-etching some samples were cleaved and their plan and crosssectional views obtained with an SEM. Some representative micrographs appear in Fig. 2 here.

\section{PhC nanofabrication with rigid stamp nanoimprint lithography}

Direct-write e-beam lithography is a serial process and is thus far too slow for use as a viable commercial patterning process. Patterning even a full 2 in. diameter wafer with PhC patterns using this technique will cost upwards of $\$ 10 \mathrm{~K}$ which is clearly unacceptable. Nanoimprint lithography (NIL) has emerged in recent years as an affordable technique for the mass replication of submicron size structures over large areas. Micro and nano optics are the areas where NIL has been especially popular and thus commercial PhC patterning has been mostly carried out with this technique. Among the advantages of NIL are relatively low cost of capital equipment and high production speed which combine to make NIL patterning very affordable [26]. We have explored the two basic variants of NIL to imprint PhC patterns on GaN LED wafers with a view to understand yield limiting factors.

In this work the following two rigid stamps NIL approaches were used.

1. Heat and pressure NIL

2. Flash imprint NIL

\subsection{Heat and pressure (HEP) NIL}

A silicon stamp was first fabricated for H\&P NIL. A clean $15 \times 15 \mathrm{~mm}$ Si substrate with a thickness of $500 \mu \mathrm{m}$ was spin coated with HSQ. An inverse design of PhQC was transferred to
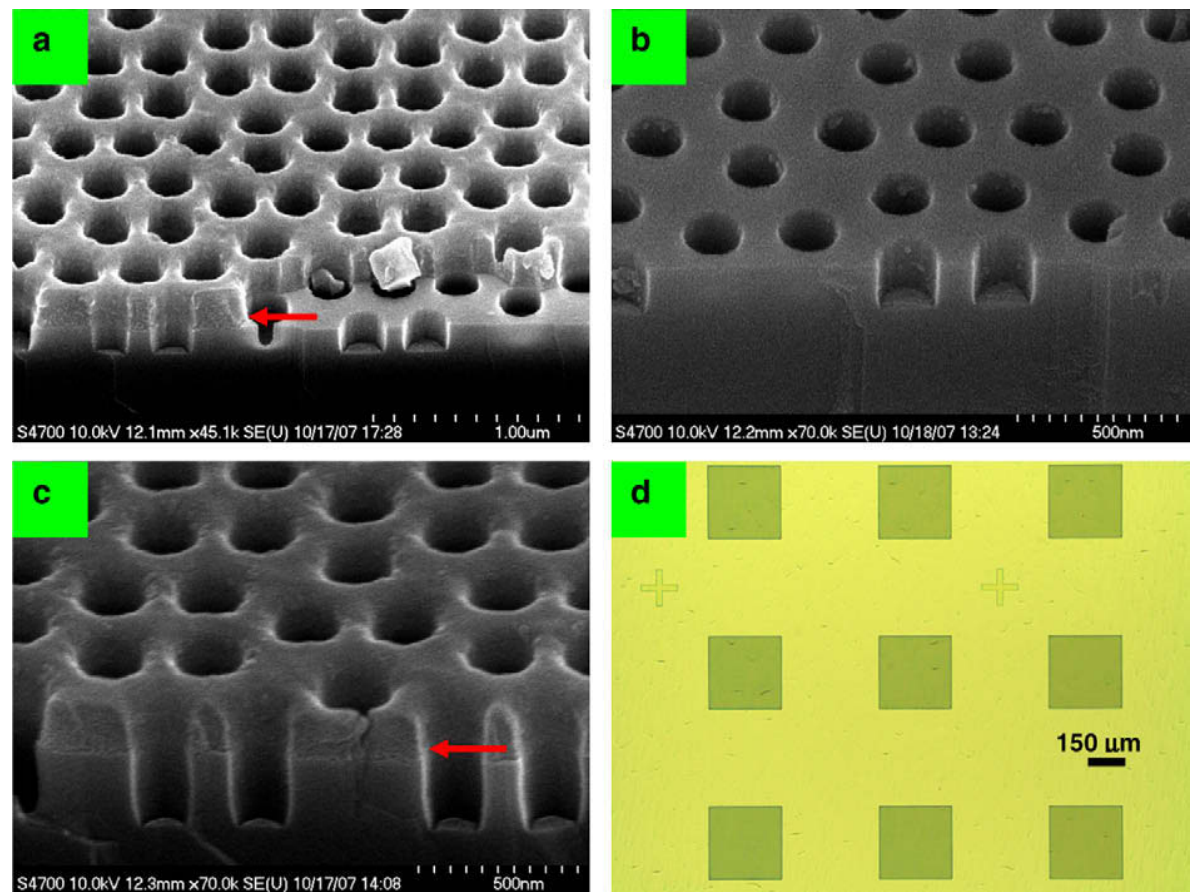

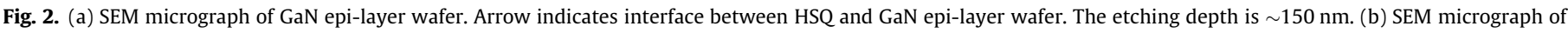

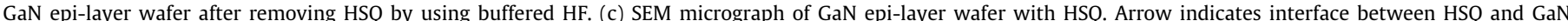

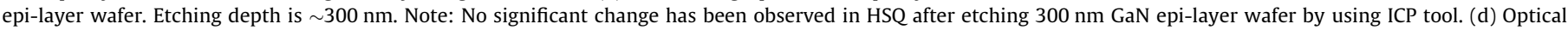
micrograph of GaN epi-layer wafer with $300 \times 300 \mu \mathrm{m}$ patterns with alignment markers. 
HSQ by using e-beam lithography. The design was further transferred to the Si substrate by inductively coupled plasma (ICP) dry-etching [27]. The gas used for dry-etching was $\mathrm{SF}_{6} / \mathrm{C}_{4} \mathrm{~F}_{8}$. The dry-etching depth was found to be $260 \mathrm{~nm}$ as measured under SEM.

This work used a commercial nanoimprint tool called nanoimprint from Obducat in Sweden. The commercially available NIL polymer mr-I-9020 [28] was spin coated on GaN epi-layer wafer at 2000 RPM. The Si stamp was placed face down on the GaN epi-layer wafer. The entire assembly was then placed in the nano-imprinter. The glass transition temperate $\left(T_{g}\right)$ of mr-I-9020 is above $100{ }^{\circ} \mathrm{C}$. The temperature was raised above the $T_{g}$ to $120^{\circ} \mathrm{C}$ and a pressure of $40 \mathrm{Bar}$ was applied. At high temperatures the viscosity of the polymer is low enough to fill all the cavities in the stamp. After a few minutes the polymer started cross-linking and this caused an increase in viscosity. The polymer solidified and the shape formation of the imprint finished after $15 \mathrm{~min}$. The stamp was detached from the GaN LED epi-layer wafer at a temperature below the $T_{g}$ of mr-I-9020. During the H\&P NIL process, pressure was used to keep the Si stamp attached with the substrate during the stamp cavity filling process.

After imprinting the GaN LED epi-layer wafer was examined under SEM. Very encouraging results were achieved with H\&P NIL, as seen in Fig. 3. It was found that this NIL technique could be used to imprint on smooth as well as on rough surfaces. The disadvantage of H\&P NIL technique is that, due to its slow speed, it cannot be used for industrial production and for processes that need alignment of the stamp with the substrate.

\subsection{Flash NIL}

Flash NIL requires UV exposure for cross-linking the NIL resist. For this reason, a quartz stamp was prepared for flash NIL. In this work a quartz substrate having dimensions of $65 \times 65 \mathrm{~mm}$ and thickness of $6 \mathrm{~mm}$ was used. NEB31 negative tone e-beam resist was spin coated on quartz substrate. NEB31 mask has an advantage over HSQ in that its etch rate is lower during quartz etching. An Inverse PhQC pattern with pillars of $550 \mathrm{~nm}$ diameter was written on
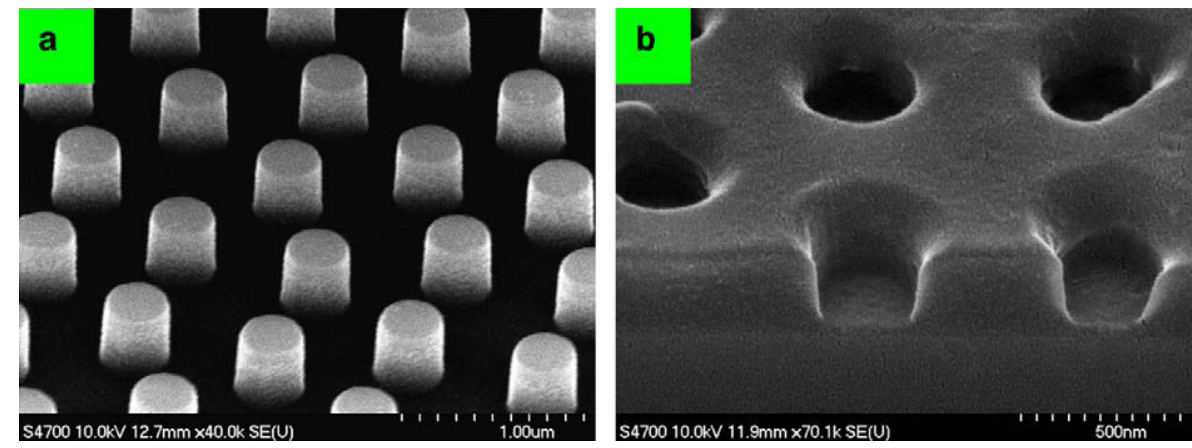

Fig. 3. (a) SEM micrograph of silicon stamp after dry-etching by using ICP tool. HSQ was removed by using buffered HF. (b) SEM micrograph of pattern imprint patterns on GaN epi-layer by using an Obducat nanoimprint imprinter tool.
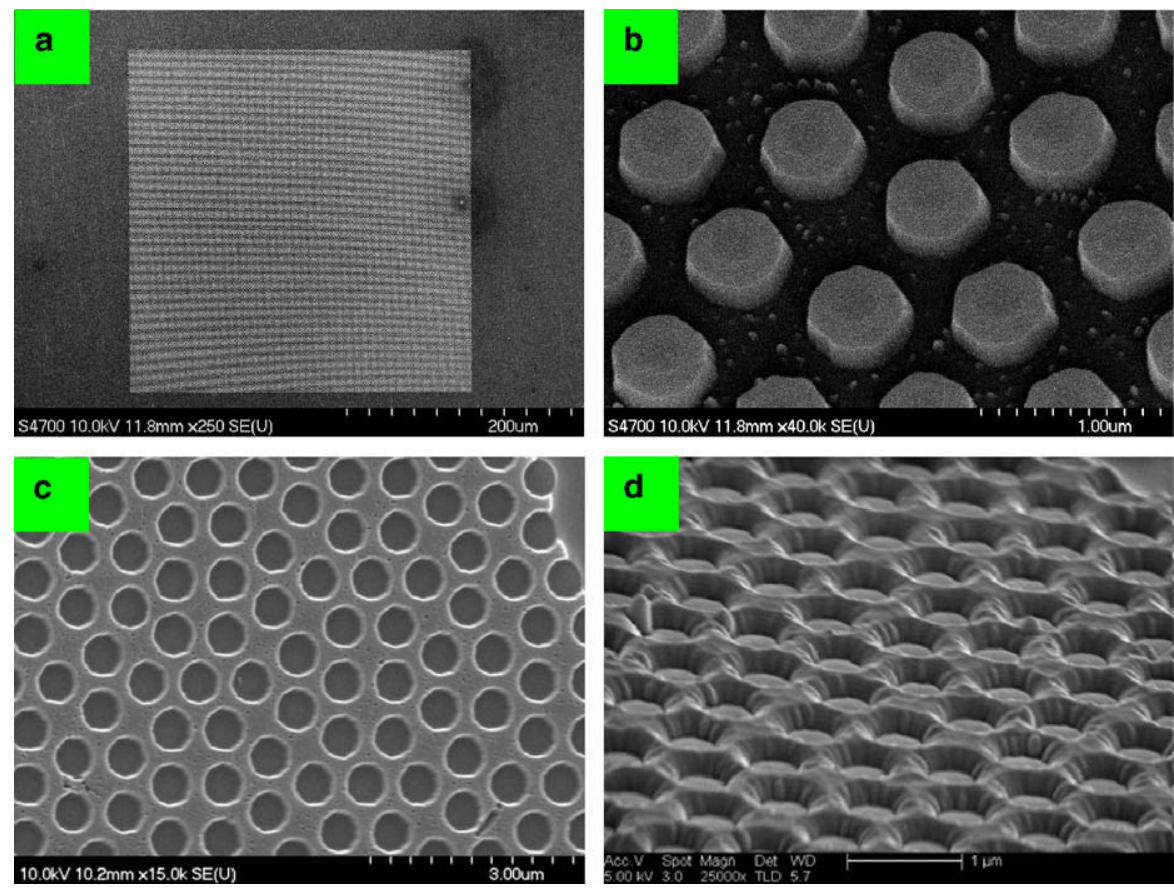

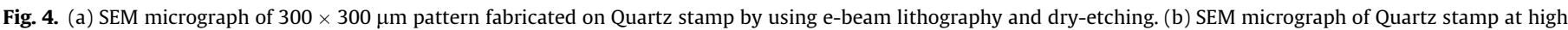

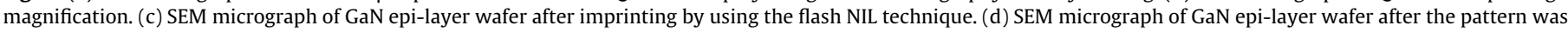
transferred into GaN by using the ICP tool. 
NEB31 resist. BP80 dry etch machine and $\mathrm{CHF}_{3}$ gas were used for dry-etching the quartz substrate. After dry-etching the patterns were examined under SEM. Vertical etching profile with depth of $180 \mathrm{~nm}$ was found in SEM.

In this work the commercially available flash NIL polymer mr-UVCUR06 [29] was spin coated on GaN epi-layer wafer at $3000 \mathrm{RPM}$. After spinning the sample was baked at $90{ }^{\circ} \mathrm{C}$ for $60 \mathrm{~s}$. Flash NIL was done by using the hard contact mode of an MA6 mask aligner. The sample was exposed to UV for $5 \mathrm{~min}$. After flash NIL, the substrate was cleaved and observed under SEM. It was found that the thickness of the residual layer was $20 \mathrm{~nm}$ whereas the NIL feature depth was $180 \mathrm{~nm}$. SEM micrographs are shown in Fig. 4.

\section{PhC nanofabrication with flexible stamp nanoimprint lithography}

Rigid stamp nanoimprint lithography has its limitations. Frequently encountered problems include release from the master, stitching errors in the step and repeat process, damage to bowed wafers, especially in high-pressure imprint processes and clearing the inevitable residual layer when device fabrication involves pattern transfer into the underlying substrate. An alternative waferscale nanoimprint process is to use flexible stamp nanoimprint lithography [30-32]. In principle this nanoimprint technique reduces or even overcomes the limitations of other methods and lends itself to large scale low cost roll-to-roll processing [33]. The technique exploits the concept of a single use disposable master. It also relies upon the development of imprint resists with low surface energy that allow easy release of the disposable master. Imprint resists can be formulated to have sufficient etch selectivity to be compatible with deep relief pattern transfer into gallium nitride substrates.

Master structures can be made using a variety of techniques, such as photolithography in photoresist or direct-write electron

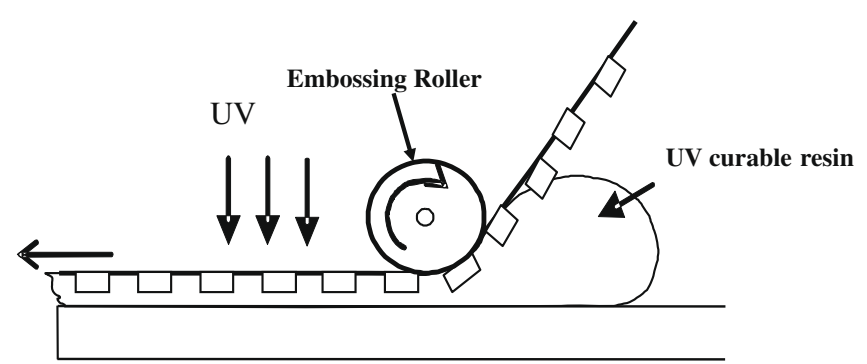

Fig. 5. Schematic of pattern transfer with a disposable master film. A roller is shown pressing the patterned film into a UV-curable polymer layer on a harder substrate. Photo-curing of the imprinted polymer allows clean separation of the master. beam patterning and then transferred to nickel replicas by standard electroforming techniques. The pattern on the nickel can then be transferred to the surface of a polyethylene terephthalate (PET) film using a roll-to-roll UV replication process. This provides a large supply (100's of metres) of "disposable masters" for nanoimprinting.

The UV cross-linking materials used in the replication process comprises a conventional acrylate system containing a small percentage of fluoroacrylate. The formulation of each resist can be designed so that spin coated films have a reduced surface energy enabling clean release of the master without additional processing steps. The disposable masters are flexible; their stiffness is determined by the combination of the PET backing film and the formulation of the lacquer containing the relief.

The first step in the process is to spin coat the substrate (typically gallium nitride) with a thin film of UV sensitive imprint resist, followed by a short low temperature pre-bake to evaporate excess solvent and to promote adhesion of the polymer film to the wafer. The imprint step involves using a roller to press the disposable master into the resist with only light force, as shown schematically in Fig. 5. Unlike conventional imprinting, the line of contact of the flexible master makes contact pressure virtually irrelevant. In many cases a simple hand roller is fit for purpose. Experiments show that the degree of filling and the thickness of the residual layer are controlled by the thickness of the spin coated layer rather than the pressure.

After imprinting the next step is to cure the resist. This involves a short exposure to UV light and an optional thermal cure to complete the cross-linking of the imprint resist. Finally the disposable master is released by peeling it from the imprinted substrate. It is often found that the disposable master is already partially released after the cure, owing to the low surface energy design of the imprint resists, eliminating the need for force at a critical stage. Fig. 6 shows a pattern that has been transferred to a wafer using this process. The pattern consists of an array of 400 diameter nm holes, shown at the right. The left hand panel in this figure shows the master stamp used in this process. It was directly written onto silicon by electron beam lithography.

Given that disposable masters are produced on a thin PET film, in a roll-to-roll process, they are highly cost effective to produce. A single rigid master structure can be used to produce many 100 's or 1000 's of metres of disposable master film. As implied by the description, the individual disposable master films are cheap enough to be discarded after each use. This provides a distinct advantage over rigid stamps, which are more expensive to produce, as they are made from more expensive materials (metals, silicones) which also need to be much thicker to provide the required rigidity and robustness.

There are, however, several issues that can limit the applicability of flexible master nanoimprint lithography. Firstly, if the pat-
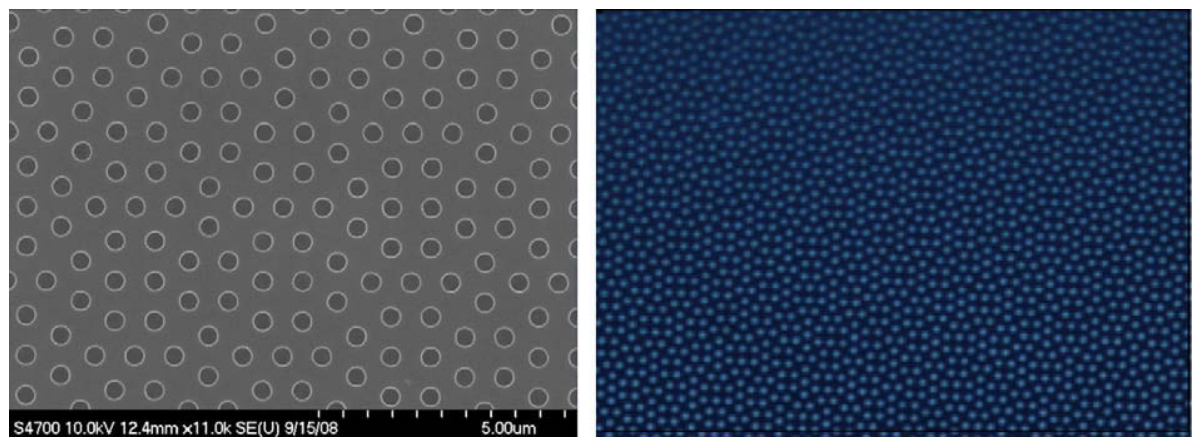

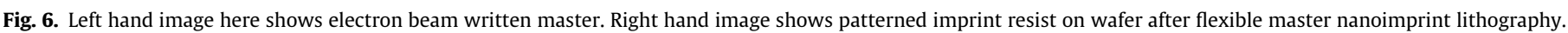


tern is to be successfully transferred to a GaN wafer by means of etching then the "residual layer", or layer of imprint resist in between the pattern and the wafer, needs to be as thin as possible. This implies excellent control over imprint resist spin coating and may impact the choice of materials that can be used in the imprint resist formulation. Secondly, the imprint resist must be formulated to provide the appropriate etch selectivity. The choice of materials available to impart etch resistance may not correspond with the requirements for achieving a thin residual layer. We have successfully explored the use of nano-silica-loaded NIL resist in this work for imparting increased etch resistance to patterned resist. Special resist formulations containing an optimum amount of nano-silica can significantly reduce resist erosion during dryetching and thus provide a higher etch selectivity relative to GaN. This is distinct from the incorporation of oxetanyl xilsesquioxane (OXSQ) units that contain molecular scale $\mathrm{Si}-\mathrm{O}$ cage units [33]. In our work we made use of much bigger ( $\sim 40 \mathrm{~nm}$ diameter) nano-silica particles that resulted in etch rates down to $50 \mathrm{~nm} / \mathrm{min}$ i.e. about a factor of four lower than the GaN etch rate under the same conditions Thirdly, the flexible master must be manufactured from thin PET to maintain flexibility and the UV replicated coating containing the relief must not limit the flexibility. This implies a thin replicated coating and therefore can restrict the use of deeper surface relief profiles.

\section{Dry-etching for pattern transfer}

Inductively coupled plasma (ICP) etching is now used in most fabrication of GaN-based LEDs [34]. This process offers high-density plasmas with concentrations of reactive species in the $10^{12} \mathrm{~cm}^{-3}$ range, and also gives wide scope for manipulating the physical and chemical contributions to the overall etch process. In standard ICP processing of GaN LED structures grown on insulating sapphire substrates, etching proceeds through the $p$-type layers and active region to produce mesas. Control of the etch depth and sidewall profile are relatively non-critical in this application. However, in the fabrication of PhC and PhQC LEDs, ICP etching has a second, more demanding role in transferring the photonic microstructure from a mask material into the GaN itself. The examples in this paper all involve pattern transfer into the topmost $p$-type GaN layers of LED structures grown on $c$-plane sapphire by metal organic vapour phase epitaxy. In this situation, the GaN is etched on the gallium-polar $\left(\begin{array}{llll}0 & 0 & 0 & 1\end{array}\right)$ crystal plane of the wurtzite-phase material. Processing of flip-chip and vertical-current LEDs

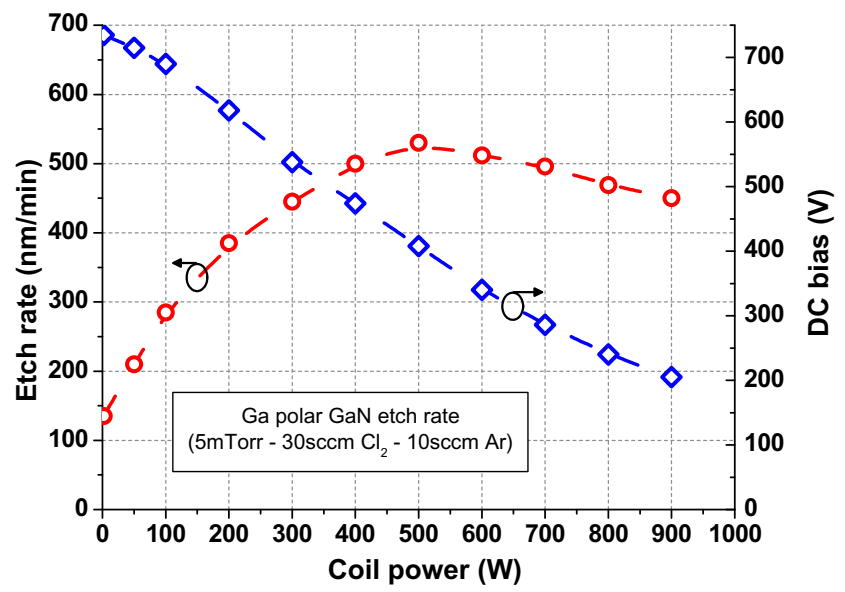

Fig. 7. Etch rate on the GaN $\left(\begin{array}{llll}0 & 0 & 0 & 1\end{array}\right)$ crystal face and dc self-bias as a function of ICP coil power. Other process parameters were kept constant, and are detailed in the text. mentioned in the introduction involves dry-etching of GaN on the nitrogen-polar $\left(\begin{array}{llll}0 & 0 & 0 & \overline{1}\end{array}\right)$ crystal plane, which may show dissimilar behaviour with respect to both etch rate and roughening $[35,36]$. The need for separate optimisation of such processes has not been widely recognised in the literature.

The ICP etch tool used in work at Strathclyde University is a Surface Technology Systems LPX system, which is equipped with a laser reflectometer. Chlorine-argon gas mixtures were used, and samples were mounted with grease or wax on silicon carrier wafers. For routine etching of LED mesas, ICP etch processes are operated at etch rates often in the $500-1000 \mathrm{~nm} / \mathrm{min}$ range. However, considerably slower etch rates are desirable for accurate control of PhC and PhQC hole depths. More specifically, the total process time should be long compared to the stabilisation time of a few seconds expected after the plasma is first struck. Etch rates may be controlled effectively by adjusting the inductively-coupled coil power; with zero coil power the process becomes equivalent to a simple capacitively-coupled reactive ion etch process. Fig. 7 shows the dependence between etch rate on the GaN ( $\left.\begin{array}{llll}0 & 0 & 0 & 1\end{array}\right)$ face and coil power, holding other process parameters constant. These included a platen power of $200 \mathrm{~W}$, a pressure of 5 milliTorr, a chlorine flow of 30 standard cubic centimetres per minute $(\mathrm{sccm})$, and argon flow of $10 \mathrm{sccm}$, and a chuck set point temperature of $30{ }^{\circ} \mathrm{C}$. The recipe with a coil power of $500 \mathrm{~W}$ is representative of one used for mesa etching in standard LED processing. The recipe with a $50 \mathrm{~W}$ coil power, corresponding to an etch rate in the plot of $210 \mathrm{~nm} / \mathrm{min}$, was chosen for the etching of PhQC structures.

HSQ masks patterned by e-beam lithography, as discussed in Section 2, proved satisfactory for the transfer of PQC patterns into GaN. The etch selectively between HSQ and GaN is similar to the value of $1: 6$ obtained between conventional $\mathrm{SiO}_{2}$ hard masks and GaN. Etch rates measured from profiling of long step features also transferred accurately to sub-micron HSQ patterns. Fig. 8 shows a representative atomic force microscope (AFM) images of a PhQC test patterns etched to a nominal hole depth of $105 \mathrm{~nm}$. The measured hole depth on this sample, averaged from five locations, was $99 \mathrm{~nm}$. A second sample etched to a target hole depth of $55 \mathrm{~nm}$ was also examined by AFM. The measured hole depth, averaged from three locations, was $56 \mathrm{~nm}$.

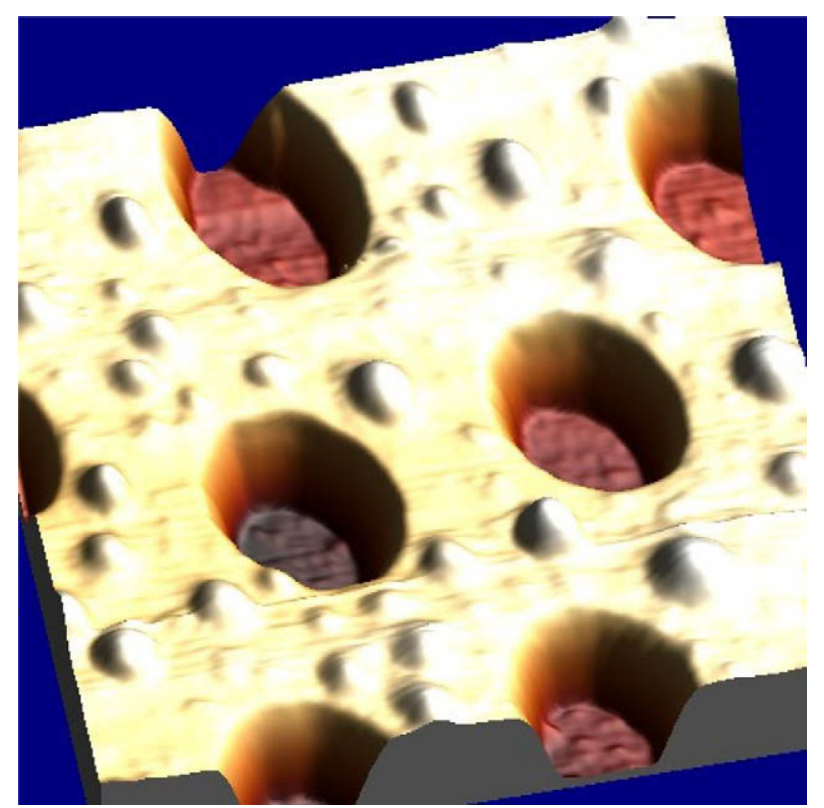

Fig. 8. Tapping-mode AFM image of $2 \times 2 \mu \mathrm{m}$ area of a PQC test pattern etched into GaN, with a hole diameter of $400 \mathrm{~nm}$, and nominal hole depth of $105 \mathrm{~nm}$. The protrusions on the surface surrounding the holes are believed to originate during epitaxial growth. 
Fig. 7 also shows the trend in dc self-bias with changing coil power, which is relevant to the sidewall slope obtained in ICP etching. The larger dc biases at lower coil powers enhance the sputter-based physical contribution, and thus favour formation of near-vertical sidewalls, if other factors are favourable. However, the actual sidewall slope obtained is also a function of the HSQ mask profile, cross-sectional SEM views of which are shown in Fig. 2. Experimental determination of the sidewall slope of PhQC holes in GaN of depths of interest for working devices (typically $<150 \mathrm{~nm}$ ) is challenging. However, cross-sectional SEM views of a specially prepared test sample with a hole depth of $800 \mathrm{~nm}$ showed that the GaN sidewalls are within $10^{\circ}$ of vertical with the preferred etch recipe.

As noted already, the ICP etch tool employed features a laser reflectometer allowing real-time in situ monitoring of the etch process through thin film interference effects. This monitoring technique is now very common in epitaxial growth, but is also widely adopted for endpoint detection in plasma etch systems [37]. The monitoring wavelength in this study was $670 \mathrm{~nm}$, and the beam was focussed onto the sample at normal incidence through a window in the ceiling of the process chamber. The refractive index difference of $\sim 0.6$ between GaN and sapphire substrates produces large changes in sample reflectance as the optical thickness of the GaN layer changes. Derivation of GaN etch rates is thus very straightforward, and is analogous to the already widespread use of reflectometry in growth monitoring [38]. Provided the GaN layer thickness is sufficiently large, etch rates under several different etch conditions can be measured in a single process run. This methodology was used to obtain the data plotted in Fig. 7, using a uniform GaN epi-layer of nominal $4 \mu \mathrm{m}$ thickness. The ternary alloy layers of LED structures, as illustrated in Fig. 1, do not greatly complicate reflectance monitoring per se, since their refractive indices do not differ from that of GaN by more than a very few percent. However, etch rates of aluminium-containing alloys are lower than those of GaN, and thus simple GaN epi-layers are preferred to full LED structures for studies of GaN etch rates.

Reflectance monitoring also allows etch rates of novel mask materials, and the selectivities with respect to GaN etch rates, to be determined in a straightforward fashion. These measurements are particularly important for polymeric nanoimprint resists, where the finite thickness of the residual layer needs to be taken into account for pattern transfer into the underlying GaN. The polymer formulations themselves have high transparency in the visible, and a suitable sample type to study the etch resistance is an unpatterned layer of the polymer on an opaque silicon substrate. Illustrative results are shown in Fig. 9, which is a plot of reflectance versus time during exposure of a 230-nm layer of a cured acrylate-fluoroacrylate resin, as discussed in Section 4, to the etch conditions used for pattern transfer into GaN. Full removal of the polymer is evidenced by the constant reflectance value reached after at etch time of $\sim 25 \mathrm{~s}$. The flat reflectance signal in the first $2 \mathrm{~s}$ after the process start corresponds to a 'dead time' caused by plasma stabilisation. The effective etch time of $23 \mathrm{~s}$ for the polymer layer thus corresponds to an average etch rate of $600 \mathrm{~nm} / \mathrm{min}, \sim 3 \times$ that of GaN under the same conditions. Even this relatively low etch resistance proved sufficient for transfer of submicron motheye-type patterns [39], produced by the flexible stamp nanoimprint lithography method discussed in Section 4, into GaN layers. However, much lower polymer etch rates down to $50 \mathrm{~nm} / \mathrm{min}$ have been demonstrated using modified resins employed in the most recent work on PQC-LED fabrication. That work shows that using proprietary blends of acrylate-fluoroacrylate resin the ICP etch rate of the resist can be reduced from $3 \times$ that of GaN to less than that of GaN. While resist without nano-silica can be successfully used for the transfer of shallow patterns to GaN, silica-loaded resists can enable the transfer of significantly deeper patterns.

After pattern transfer, LED structures measuring $350 \times 350 \mu \mathrm{m}$ were fabricated using standard photolithography, dry-etching and metallization techniques. An SEM micrograph of our finished PhQC LED device appears in Fig. 10. The $n$-contact pad, seen to the right, required etching down to the $n$-type contact layer of the epitaxial

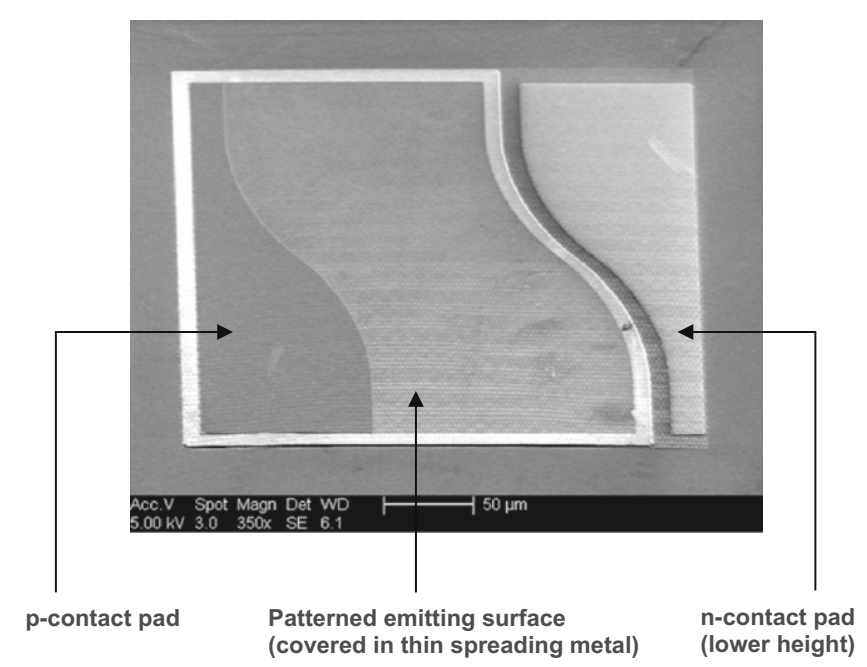

Fig. 10. SEM micrograph of a finished PhQC LED device showing the central area patterned with PhQC structure as well as the $n$ and $p$ contacts to the right and left, respectively.

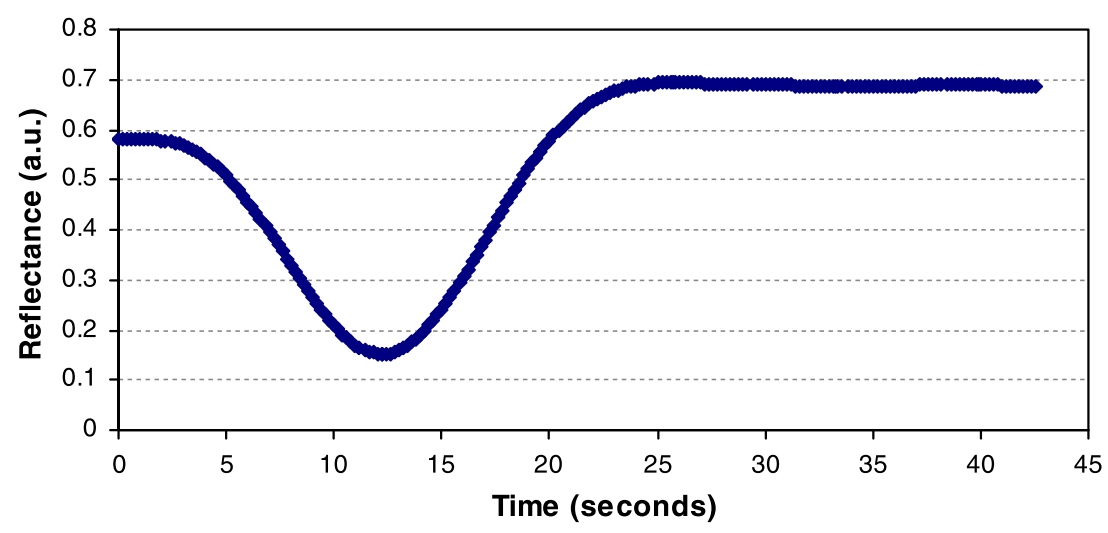

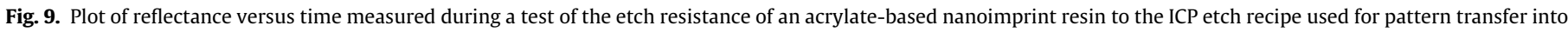
GaN. 
structure whereas the $p$-contact pad was formed directly on top of the wafer. A thin Ni/Au bilayer spreading metal was deposited on the top face of the LED to distribute current evenly across the entire cross-section of the device. This transparent conductive layer is needed in all large area LEDs because the relatively low electrical conductivity of $p$-type GaN would otherwise lead to current crowding effects resulting in uneven illumination from the emitting face of the LED. In order to further reduce current crowding effects and resulting non-uniform thermal effects in the LED chip we utilised anode and cathode electrode designs developed by Bulashevich and colleagues from simulation of such effects in blue GaN LEDs [40]. The curved contours of this design are clearly seen in Fig. 10. We tested our devices both on wafer and after dicing and bonding onto TO-style headers. Their I-V characteristics are essentially identical to unpatterned reference devices made on the same chips, thus showing that the PhQC patterning process doesn't cause electrical degradation of LEDs. These devices performed very well in our tests, generating strong and uniform emission from the top emitting face. Detailed descriptions of our electrical and optical tests on these devices will be published elsewhere.

\section{Summary}

We have described nanofabrication technologies for patterning photonic crystal structures on LED wafers. These include directwrite electron beam lithography, hard stamp nanoimprint lithography, soft-stamp nanoimprint lithography for patterning resist and inductively-coupled plasma-based dry-etching for transferring patterns into semiconductor material. Process details have been provided which clearly show that electron beam lithography is desirable for R\&D purposes whereas nanoimprint lithography is the only option for large scale device manufacturing. Soft-stamp nanoimprint lithography with disposable masters, in particular, appears to be the lowest cost technique for mass manufacture of PhC LEDs. ICP dry-etching is also demonstrated as ideal for transferring patterns defined in resist to the underlying semiconductor wafer material with high fidelity.

\section{Acknowledgements}

The authors would like to thank the UK Government Technology Strategy Board (TSB), formerly known as the Department of Trade and Industry (DTI), for financial support of the photonic quasi-crystals for Display Illumination (PQLDI) project, under grant No. K2506L. Thanks are also due to all technical staff at the University of Glasgow's James Watt Nanofabrication Centre (JWNC) as well as Benoit Guilhabert and Yanfeng Zhang at the University of Strathclyde's Institute of Photonics. We also acknowledge help from Haoxiang Zhang in relation to the LED electrode structure employed in this work. The authors would also like to thank Majd Zoorob and Tom Lee for technical support and useful discussions during the early stages of the project.

\section{References}

[1] Gilbert Held, Introduction to Light Emitting Diode Technology and Applications, Auerbach Publications, Boca Raton, 2008, ISBN: 1420076620.

[2] Arturas Zukauskas, Michael S. Shur, Remis Caska, Introduction to Solid-State Lighting, John Wiley and Sons Inc., New York, 2002, ISBN: 0-471-21574-0.

[3] Michael R. Krames, Oleg B. Shchekin, R. Mueller-Mach, G.O. Mueller, Ling Zhou, M. George Craford, Journal of Display Technology 3 (2007) 160-174.
[4] Gerard Harbers, Serge J. Bierhuizen, Michael R. Krames, Journal of Display Technology 3 (2007) 98-108.

[5] Christopher Wiesmann, Krister Bergenek, Norbert Linder, Ulrich T. Schwarz, Laser and Photon Review 3 (2009) 262-286.

[6] Chul Huh, Kug-Seung Lee, Eun-Jeong Kang, Seong-Ju Park, Journal of Applied Physics 93 (2003) 9383-9386.

[7] T. Mukai, S. Nagahama, T. Yamamoto, M. Sano, D. Morita, M. Yamamoto, M. Nonaka, K. Yasutomo, K. Akaishi, Physica Status Solidi C 2 (2005) 3884-3886

[8] K. Tadatomo, H. Okagawa, Y. Ohuchi, T. Tsunekawa, Y. Imada, M. Kato, T. Taguchi, Japanese Journal of Applied Physics 40 (2001) L583.

[9] T.N. Oder, J. Shakya, J.Y. Lin, H.X. Jiang, Applied Physics Letters 83 (2003) 1231

[10] Hyun Kyong Cho, Junho Jang, Jeong-Hyeon Choi, Jaewan Choi, Jongwook Kim, Jeong Soo Lee, Beomseok Lee, Young Ho Choe, Ki-Dong Lee, Sang Hoon Kim, Kwyro Lee, Sun-Kyung Kim, Yong-Hee Lee, Optics Express 14 (2006) 86548660.

[11] Aurélien David, Tetsuo Fujii, Rajat Sharma, Kelly McGroddy, Shuji Nakamura Steven P. DenBaars, Evelyn L. Hu, Claude Weisbuch, Henri Benisty, Applied Physics Letters 88 (2006) 0611241-0611243.

[12] Faiz Rahman, Richard De La Rue, Photonics Spectra 41 (2007) 52-55.

[13] P.R. Tavernier, D.R. Clarke, Journal of Applied Physics 89 (2001) 1527-1536.

[14] Jonathan J. Wierer Jr., Aurélian David, Mischa M. Megens, Nature Photonics 3 (2009) 163-169.

[15] K. McGroddy, A. David, E. Matioli, M. Iza, S. Nakamura, S. DenBaars, J.S. Speck C. Weisbuch, E.L. Hu, Applied Physics Letters 93 (2008) 1035021-1035023.

[16] M.D.B. Charlton, M.E. Zoorob, T. Lee, Proceedings of SPIE 6486 (2007) 64860R1-64860R10.

[17] Aurélian David, Henri Benisty, Claude Weisbuch, Journal of Display Technology 3 (2007) 133-148

[18] Michael P.C. Watts, M. Zoorob, T. Lee, J. McKenzie, Proceedings of SPIE 6462 (2007) 64620N1-64620N12.

[19] H.W. Huang, C.H. Lin, K.Y. Lee, C.C. Yu, J.K. Huang, B.D. Lee, H.C. Kuo, K.M. Leung, S.C. Wang, Semiconductor Science and Technology 24 (2009) 0850081-085008-5.

[20] J. Soellner, O. Schoen, A. Alam, B. Schineller, J. Kaeppeler, M. Heuken, Thin Solid Films 515 (2007) 4372.

[21] J. Li, J.Y. Lin, H.X. Jiang, Applied Physics Letters 88 (2006) 171909-1-171909-3.

[22] M.E. Zoorob, M.D.B. Charlton, G.J. Parker, J.J. Baumberg, M.C. Netti, Nature 404 (2000) 740-743

[23] Keunjoo Kim, Jaeho Choi, Jong Bae Park, Sang Cheol Jeon, Jin Soo Kim, Hee Mok Lee, IEEE Photonics Technology Letters 20 (2008) 1455.

[24] B. Rong, R. Cheung, W. Gao, M. Kamp, Microelectronic Engineering 53 (2000) 419-422.

[25] Philip A. Shields, Martin D.B. Charlton, Tom Lee, Majd E. Zoorob, Duncan W.E. Allsopp, Wang N. Wang, IEEE Journal of Selected Topics in Quantum Electronics 15 (2009) 1269-1274.

[26] Sang Hoon Kim, Ki-Dong Lee, Ja-Yeon Kim, Min-Ki Kwon, Seong-Ju Park, Nanotechnology 18 (2007) 055306-055311.

[27] V.A. Yunkin, D. Fischer, E. Voges, Microelectronic Engineering 27 (1995) 463 466.

[28] K. Pfeiffer, F. Reuther, M. Fink, G. Gruetzner, P. Carlberg, I. Maximov, L. Montelius, J. Seekamp, S. Zankovych, C.M. Sotomayor-Torres, H. Schulz, H.-C. Scheer, Microelectronic Engineering 67-68 (2003) 266-273.

[29] Marko Vogler, Sabine Wiedenberg, Michael Muhlberger, Iris Bergmair, Thomas Glinsner, Holger Schmidt, Ernst-Bernhard Kley, Gabi Grutzner, Microelectronic Engineering 84 (2007) 984-988.

[30] L.J. Guo, Advanced Materials 19 (2007) 495-513.

[31] JaeJong Lee, SooYeon Park, KeeBong Choi, GeeHong Kim, Microelectronic Engineering 85 (2008) 861-865.

[32] Se Hyun Ahn, L. Jay Guo, Advanced Materials 20 (2008) 2044-2049.

[33] G. Hubbard, S.J. Abbott, Q. Chen, D.W.E. Allsopp, W.N. Wang, C.R. Bowen, R. Stevens, A. Satka, D. Hasko, F. Uherek, J. Kovac, Physica E: Low-dimensional Systems and Nanostructures 41 (2009) 1118-1121.

[34] K.H. Baik, S.J. Pearton, Applied Surface Science 255 (2009) 5948-5951.

[35] F. Rizzi, E. Gu, M.D. Dawson, I.M. Watson, R.W. Martin, X.N. Kang, G.Y. Zhang, Journal of Vacuum Science \& Technology, A: Vacuum, Surfaces, and Films 25 (2007) 252-260

[36] I. Waki, M. Iza, J.S. Speck, S.P. DenBaars, S. Nakmuara, Japanese Journal of Applied Physics 45 (2006) 720-723.

[37] I.P. Herman, Annual Review of Physical Chemistry 54 (2003) 277-305.

[38] C. Liu, I.M. Watson, Semiconductor Science and Technology 22 (2007) 629635.

[39] Hideki Kasugai, Yasuto Miyake, Akira Honshio, Shunsuke Mishima, Takeshi Kawashima, Kazuyoshi Iida, Motoaki Iwaya, Satoshi Kamiyama, Hiroshi Amano, Isamu Akasaki, Hiroyuki Kinoshita, Hiromu Shiomi, Japanese Journal of Applied Physics 44 (2005) 7414-7417.

[40] K.A. Bulashevich, I.Yu. Evstratov, V.F. Mymrin, S. Yu. Karpov, Physica Status Solidi C 4 (2007) 45-48. 